\title{
Breadth-First Maximum-Likelihood Sequence Detection of Ring Convolutional Coded Continuous Phase Modulation
}

\author{
ZHANG Lei $^{1, a^{*}}$, LU Huaping ${ }^{1}$, ZHU Aimin ${ }^{1}$ and CUI Jiuqiang ${ }^{1}$ \\ 'Zhenjiang Watercraft College, Zhenjiang, China \\ ajasilet@163.com
}

\begin{abstract}
Keywords: Continuous phase modulation; ring convolutional codes; maximum-likelihood sequence detection; breadth-first.

Abstract. This paper shows a breadth-first maximum-likelihood sequence detection method for ring convolutional coded continuous phase modulation (CPM) over additive white Gaussian noise (AWGN) channel. By reserving the states which are closest to the received signal in the normalized squared Euclidean distance after every state has only one best path the high detection complexity is reduced according to our needs. Numerical results shows that this detection method maintains good performances comparing with the maximum-likelihood detector based on Viterbi algorithm(VA) with a significant reduction in detection complexity.
\end{abstract}

\section{Introduction}

Continuous phase modulation (CPM) is a bandwidth and power efficient modulation scheme which shows a great prospective in future communication systems and networks $[1,2,3]$. The concept of tilted phase which makes the trellis diagram to be time-invariant is introduced to reduce the detection complexity of the time-variant trellis diagram of CPM signal [4]. With tilted phase the CPM signal is decomposed into an inner encoder and a memoryless modulation (MM), and the inner encoder is called continuous phase encoder (CPE) since it provides CPM with continuous phase capability. CPE can be viewed as a convolutional encoder over rings, and the concept of ring convolutional codes is first introduced to be used in the $M$-ary phase modulation systems by Massey and Mittelholzer[5]. Trellis-coded continuous phase modulation schemes based on convolutional codes over rings are developed in [6-9]. Due to the memory property of joint trellis in the ring convolutional coded CPM signals, Viterbi Algorithm (VA) is used to performance maximum likelihood sequence detection when the channel is additive white Gaussian noise (AWGN). The number of states in the joint trellis of the signal grows exponentially with the constraint length of the overall encoder.

For signals with trellis property, breadth-first approach is taken by Viterbi in [10] for decoding of convolutional codes. Reduced state sequence detection for trellis signals are considered in $[11,12,13]$. In [14], reduced state sequence detection (RSSD) which combines decision feedback with Viterbi decoding for full response CPM is studied, and then RSSD for partial response CPM is also considered in [15]. In [16, 17], Aulin shows the breadth-first maximum-likelihood sequence detection (MLSD) algorithm $\mathrm{SA}(B, C)$ and provides the performance analysis. The $\mathrm{SA}(B, 1)$ which is known as $M$-algorithm is used to detect CPM signal over AWGN and multipath channels [18]. The dropping procedure of the algorithm faces the problem of wasting space when the some of best paths reserved are merging into the same state. So, this paper considers dropping the paths merging into the same state with lager Euclidean distance firstly. The algorithm is presented as $\mathrm{SA}(1, C)$ where the $C$ partitions of states are variable.

The paper is organized as follows. Firstly, ring convolutional coded CPM is described using the Rimoldi approach in succeeding Section. Then, the details of the $\operatorname{SA}(1, C)$ are given. And then, the performance for AWGN channel is shown using computer simulation. At last, some conclusions are given. 


\section{Ring Convolutional Coded Continuous Phase Modulation}

By using $\boldsymbol{u}$ with $u_{i} \in\{0,1, \cdots, M-1\}$ to represent the input symbols of $M$-ary CPM signal, CPM signal without feedback is written as

$$
s(t, \boldsymbol{u})=\sqrt{2 E_{s} / T} \cos \left(2 \pi f t+\varphi(t, \boldsymbol{u})+\varphi_{0}\right), \quad t \geq 0
$$

where $E_{s}$ is the symbol energy, $T$ is the symbol period, $f$ is the carrier frequency, $\square$ is the initial phase which is usually taken as zero, and the information carrying phase is

$$
\varphi(t, \boldsymbol{u})=4 \pi h \sum_{i=0}^{+\infty}\left(u_{i}-u_{i-1}\right) q(t-i T), \quad t \geq 0
$$

where $h$ is the modulation index ( $h=q / p, q$ and $p$ are relatively prime positive integers). $q(t)$ is the phase response function which should satistified

$$
q(t)= \begin{cases}0, & t \leq 0 \\ 1 / 2, & t>L T\end{cases}
$$

where $L$ is a positive integer which is called the memory length of CPM signal.

During the $n$th symbol interval $(n T \leq t<(n+1) T)$, the physical phase is

$$
\varphi^{\prime}(t, \boldsymbol{u})=R_{2 \pi}\left[2 \pi h R_{p}\left[u_{n-L}\right]+4 \pi h \sum_{i=n-L+1}^{n}\left(u_{i}-u_{i-1}\right) q(t-i T)\right]
$$

whose trellis is time-invariant. In [4], $R_{p}[$.$] is the "modulo p$ operator" and $R_{2 \square}[$.$] is the "modulo$ $2 \square$ operator".

Transmitted signal during the $n$th symbol interval is depended only on vector $\boldsymbol{X}_{n}=\left[u_{n}-u_{n-1}\right.$, $\left.u_{n-1}-u_{n-2}, \cdots, u_{n-L+1}-u_{n-L}, R_{p}\left[u_{n-L}\right]\right]$ which is called the output of the CPE, and the map from $\boldsymbol{X}_{n}$ to the transmitted signal $s\left(t, \boldsymbol{X}_{n}\right)$ is defined as MM.

When we restrict on the particular scheme where $M=p$. Using $D$ to present the delay of symbol interval $T$, the information symbols is $\boldsymbol{u}(D)=u_{0} D+u_{1} D+\cdots$, the output of CPE is $\boldsymbol{X}(D)=\boldsymbol{X}_{0} D+\boldsymbol{X}_{1} D+\cdots$, and the $\mathrm{CPE}$ is $\boldsymbol{X}(D)=\boldsymbol{u}(D) \boldsymbol{C}(D)$, where $\boldsymbol{C}(D)=\left[1-D, D(1-D), \cdots, D^{L-1}(1-D), D^{L}\right]$.

Let $\mathbf{Z}_{M}$ denote the ring of integers modulo $M$, a rate b/v convolutional encoder over $\mathbf{Z}_{M}$ is passing the $M$-ary information sequence through a linear finite-state shift register. When CPM combined with ring convolutional codes, the system performances are improved because the CPE and ring convolutional codes are over the same ring. The block diagram of ring convolution coded CPM schemes is shown in Fig. 1, and the overall encoder consists of a b/v ring convolutional encoder and a CPE.

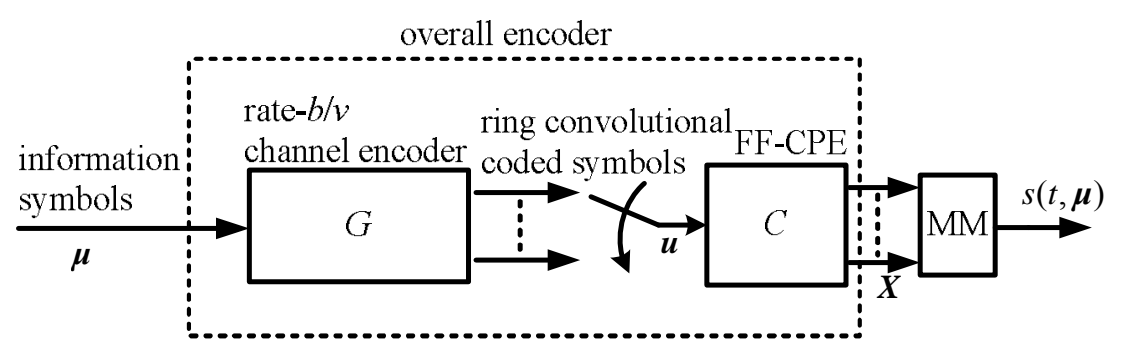

Fig. 1. Block diagram of ring convolutional coded CPM schmems.

\section{Breadth-First Maximum-Likelihood Sequence Detection}

As a subclass of the $\mathrm{SA}(\mathrm{B}, C)$ proposed in [16], a breadth-first maximum-likelihood sequence detection algorithm $\mathrm{SA}(1, C)$ is used to detect the ring convolutional coded CPM signals. Partition the 
all the $S$ elements of the sets of states for coded CPM signals into $C$ new disjoint sets which are mutually exclusive. Among the $C$ sets there are $C-1$ sets which only have one state, and the left one set includes the other $S-C+1$ states. one path that is closest to the received signal is selected for each set for every $b$ symbols. The partition for every $b$ symbols is varying according to Euclidean distance for each state to received signal.

The process of the algorithm process $\mathrm{SA}(1, C)$ is to repeat Steps 1-4 for each symbol interval.

Step 1: Extend all reserved states to the next $b$ input symbols.

Step 2: For each new state, drop all but one path that is closest to the received signal.

Step 3: Drop all new states but the $C$ states which are closest to the received signal.

Step 4: If the paths have reached the decision depth, release as output the first $b$ symbols interval of the best path.

\section{Simulation Results}

2RC CPM signals over the AWGN channel are simulated for $M=3$. The modulation index is $h=1 / 3$ according to $M=3$. Channel encoder $\left[\begin{array}{lllll}2 & 2 & 2 ; 2 & 0 & 2\end{array}\right]$ is considered. The symbol error rates of different $C$ are shown in Fig. 2. The optimal Viterbi detection (When $C$ is the same as $S=27$ ) is shown too. From the figure, it is apparently that there is only a little performance lose when $C=9$. But when $C=6$, the system performance is too bad. However, the algorithm provides us with significant reduction in complexity while maintaining good performance.

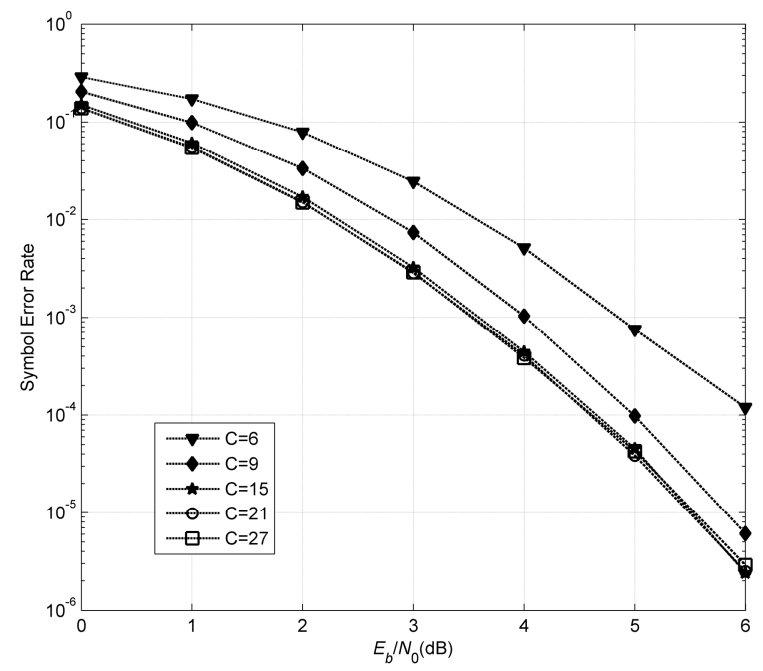

Fig. 2. Performances of symbol error rate for $M=3, h=1 / 3$ and $2 \mathrm{RC}$ CPM signals with [2 22 2;2 0 2 $]$ ] using $\mathrm{SA}(1, C)$ algotithm

\section{Summary}

The SA $(1, C)$ algorithm which performs the breadth-first maximum-likelihood sequence detection for ring convolutional coded CPM signals is suggested in this paper. Compared to Viterbi algorithm, the $\mathrm{SA}(1, C)$ algorithm provides significant reduction in complexity while maintaining good performance.

\section{References}

[1] T. Aulin and C. E. Sundberg, "Continuous phase modulation-part I: full response signaling," IEEE Trans. Commun., vol. 29, no. 3, pp. 196-209, March, 1981.

[2] T. Aulin, N. Rydbeck and C. E. Sundberg, "Continuous phase modulation-part II: partial response signaling,” IEEE Trans. Commun., vol. 29, no. 3, pp. 210-225, March, 1981. 
[3] J. B. Anderson, T. Aulin, and C. E. Sundberg, Digital Phase Modulation. New York: Plenum Press, 1986.

[4] B. Rimoldi, “A decomposition approach to CPM," IEEE Trans. Inform. Theory, vol. 34, no. 2, pp. 260-270, March, 1988.

[5] J. L. Massey and T. Mittelholzer, "Convolutional codes over rings," in Proc. 4th Joint Swedish-USSR Int. Workshop Information Theory, pp. 14-18, 1989.

[6] R. H. Yang and D. P. Taylor, "Trellis-coded continuous-phase frequencyshift keying with ring convolutional codes,” IEEE Trans. Inform. Theory, vol. 40, no. 4, pp. 1057-1067, July 1994.

[7] B. Rimoldi and Q. Li, "Coded continuous phase modulation using ring convolutional codes," IEEE Trans. Commun., vol. 43, no. 11, pp. 2714-2720, Nov. 1995.

[8] R. Maw and D. Taylor, "Space-time coded systems using continuous phase modulation," IEEE Trans. Commun., vol. 55, no. 11, pp. 2047-2051, Nov. 2007.

[9] M. Xiao and T. Aulin, "Serially concatenated continuous phase modulation with convolutional codes over rings," IEEE Trans. Commun., vol. 54, no. 8, pp. 1387-1395, Aug. 2006.

[10] A. J. Viterbi "Error bounds for convolutional codes and an asymptotically optimum decoding algorithm," IEEE Trans. Infrom. Thoery, vol. 13, pp. 260-269, April 1967.

[11] A. Duel-Hallen and C. Heegard, "Delayed decision-feedback sequence estimation," IEEE Trans. Commun., vol. 37, pp. 428-436, May 1989.

[12] P. R. Chevillat and E. Eleftheriou, "Decoding of trellis-encoded signals in the presence of intersymbol interference and noise," IEEE Trans. Commun., vol. 37, pp. 669-676, July 1989.

[13] J. B. Anderson, "Limited search trellis decoding of convolutional codes," IEEE Trans. Inform. Theory, vol. 35, pp. 944-955, September 1989.

[14] A. Svensson, "Reduced state sequence detection of full response continuous phase modulation," Electronics Letters, vol. 26, no. 10, pp. 652-654, May 1990.

[15] A. Svensson, "Reduced state scqucnce detection of partial response continuous phase modulation,” IEE Proc., vol. 138, no. 4, pp. 256-268, August 1991.

[16] T. Aulin, "Breadth-first maximum-likelihood sequence detection: Basics," IEEE Trans. Commun., vol. 47, pp. 208-216, February 1999.

[17] T. Aulin, "Breadth-first maximum-likelihood sequence detection: Geometry," IEEE Trans. Commun., vol. 51, pp. 2071-2080, June 2003.

[18] E. A. Ince, N. Vorizanakis, Y. J. Guo and S. K. Barton, "Breadth-first sequential detection of PR-CPM signals over AWGN and multipath channels," in Proceeding of ISIT 1997, Ulm, Germany, June/July 1997. 